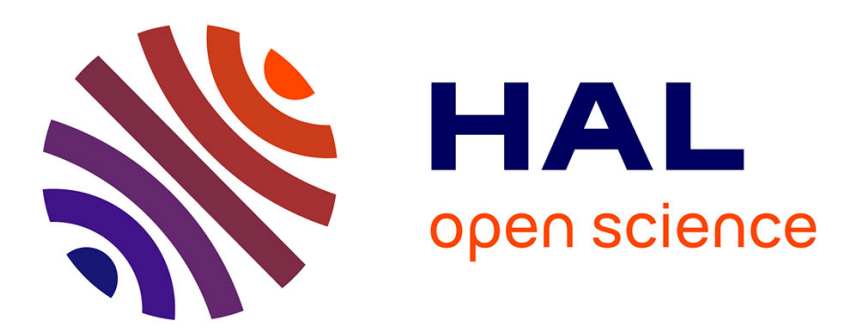

\title{
On finite Morse index solutions of two equations with negative exponent

\author{
Juan Dávila, Dong Ye
}

\section{To cite this version:}

Juan Dávila, Dong Ye. On finite Morse index solutions of two equations with negative exponent. Proceedings of the Royal Society of Edinburgh: Section A, Mathematics, 2013, 143 (1), pp.121-128. 10.1017/S0308210511001144 . hal-01095020

\section{HAL Id: hal-01095020 \\ https://hal.science/hal-01095020}

Submitted on 14 Dec 2014

HAL is a multi-disciplinary open access archive for the deposit and dissemination of scientific research documents, whether they are published or not. The documents may come from teaching and research institutions in France or abroad, or from public or private research centers.
L'archive ouverte pluridisciplinaire HAL, est destinée au dépôt et à la diffusion de documents scientifiques de niveau recherche, publiés ou non, émanant des établissements d'enseignement et de recherche français ou étrangers, des laboratoires publics ou privés. 


\title{
On finite Morse index solutions of two equations with negative exponent
}

\author{
Juan Dávila* and Dong Ye
}

\begin{abstract}
In this note, we consider the following equations involving negative exponent:$$
\Delta u=|x|^{\alpha} u^{-p}, \quad u>0 \quad \text { in } \Omega \subset \mathbb{R}^{n}
$$

and

$$
\Delta u=u^{-p}-1, \quad u>0 \quad \text { in } \Omega \subset \mathbb{R}^{n}
$$

where $p>0$. Under optimal conditions on the parameters $\alpha>-2$ and $p>0$, we prove the nonexistence of finite Morse index solution on exterior domains or near the origin. We prove also an optimal regularity result for solutions with finite Morse index and isolated rupture at 0 .
\end{abstract}

KEYWORDS: finite Morse index solution, negative exponent

AMS SUBJECT CLASSIFICATION: 35B35, 35A01, 35J60.

\section{Introduction}

Recently, many authors have studied solutions with finite Morse index for elliptic equations. For example, Farina has classified in [3] all finite Morse index classical solutions of $-\Delta u=|u|^{p-1} u$ in $\mathbb{R}^{n}$ for $1<p<p_{J L}$, where $p_{J L}$ is the Joseph-Lundgren exponent. Motivated by some models arising in engineering and physics, such as MEMS (Micro-electromechanical system) or thin films, elliptic equations with nonlinearities of negative exponent, for example $f(x) u^{-p}(p>0)$, have also drawn the attention of many works, see for instance $[2,1,4]$ and the references therein.

In this short note, we improve some results in $[1,4]$ by simple arguments, which can be also applied to similar problems with negative exponent. of

In $[1$, Theorem 1.2], the authors proved that there are no solutions with finite Morse index

$$
\Delta u=|x|^{\alpha} u^{-p}, \quad u>0 \quad \text { in } \Omega=\mathbb{R}^{n} \backslash B(0, R)
$$

for any $n \geq 2, \alpha>-2, p>p_{c}\left(\alpha^{-}\right)$and $R>0$. Here $\alpha^{-}=\min (\alpha, 0)$ and $B(x, r)$ denotes the ball of radius $r>0$ centered at $x$. Moreover, for any $\alpha>-2$, the exponent $p_{c}(\alpha)$ is given by

$$
p_{c}(\alpha)= \begin{cases}\frac{\alpha+n-\sqrt{(\alpha+2)(\alpha+2 n-2)}}{\alpha-n+4+\sqrt{(\alpha+2)(\alpha+2 n-2)}} & \text { if } 2 \leq n<10+4 \alpha \\ +\infty & \text { if } n \geq 10+4 \alpha .\end{cases}
$$

*J.D. is supported by Fondecyt 1090167, CAPDE-Anillo ACT-125 and Fondo Basal CMM.

${ }^{\dagger}$ D.Y. is supported by the French ANR project referenced ANR-08-BLAN-0335-01. The work was initiated when we both visited the East China Normal University. We would like thank the Department of Mathematics for its kind hospitality. 
For simplicity, we consider always classical solutions, i.e. $u \in C^{2}$. Let us recall that the Morse index of a solution $u$ to (1.1) is defined as the maximal dimension of all subspaces $X$ of $C_{c}^{1}(\Omega)$ such that

$$
\int_{\Omega}|\nabla \varphi|^{2} d x-p \int_{\Omega}|x|^{\alpha} u^{-p-1} \varphi^{2} d x<0, \quad \text { for all } \varphi \in X \backslash\{0\} .
$$

We say that $u$ is a stable solution to (1.1) if the Morse index is just 0 .

Going back to [1, Theorem 1.2], it is well known that $u_{0}(x)=\Lambda|x|^{\frac{2+\alpha}{p+1}}$ with

$$
\Lambda=\left[\frac{2+\alpha}{p+1}\left(n-2+\frac{2+\alpha}{p+1}\right)\right]^{\frac{-1}{p+1}}
$$

is a stable solution of (1.1) in $\mathbb{R}^{n} \backslash\{0\}$, if $\alpha>-2$ and $0<p \leq p_{c}(\alpha)$. So the situation for $\alpha>0$ and $p_{c}(\alpha)<p \leq p_{c}(0)$ was left open in [1]. Our first result is to give an answer for that.

Theorem 1.1 Assume $n \geq 2, \alpha>-2, p>p_{c}(\alpha)$ and $R>0$, then there is no solution of (1.1) with finite Morse index.

Theorem 1.1 here completes Theorems 1.1 and 1.2 in [1]. By the same idea, we get also the optimal nonexistence result for finite Morse solution of (1.1) near the origin.

Theorem 1.2 Assume $n \geq 2, \alpha>-2, p>p_{c}(\alpha)$ and $R>0$, then there is no solution of $\Delta u=|x|^{\alpha} u^{-p}, u>0$ in $B(0, R) \backslash\{0\}$ with finite Morse index that has an isolated rupture at 0.

This optimal result completes Theorem 1.3 in [1], again for the case $\alpha>0$ and $p_{c}(\alpha)<p \leq p_{c}(0)$. The solution $u$ has isolated rupture at the origin means that $\lim _{x \rightarrow 0} u(x)=0$. We define the Morse index as for (1.1), replacing just $\Omega$ by $\{0<|x|<R\}$.

As a corollary, we obtain also the following regularity result which generalizes Theorem 1.4 in $[1]$.

Theorem 1.3 Assume $n \geq 2, \alpha>-2$ and $p>0$. If $u$ is a classical solution of $\Delta u=|x|^{\alpha} u^{-p}$ in $B(0, R) \backslash\{0\}$ with finite Morse index and an isolated rupture at 0 , then $u$ is Hölder continuous at 0 . More precisely, defining $u(0)=0$, we have

$$
u \in C^{\frac{2+\alpha}{p+1}}(B(0, R)) .
$$

In [4], the authors considered the equation

$$
\Delta u=u^{-p}-1, \quad u>0 \quad \text { in } \mathbb{R}^{n} \backslash B(0, R)
$$

with $p>0$. In particular, they proved that when $p>p_{c}(0)$, no solution with finite Morse index of (1.3) exists. More precisely, $p>\max \left(p_{c}(0), \frac{(n-2)^{2}}{8 n}\right)$ was required, but we can check easily that the maximum is just $p_{c}(0)$. Consequently, Theorem 1.3 in [4] had sense only for $n<10$ since $p_{c}(0)=\infty$ if $n \geq 10$.

Here we want to point out that the problem (1.3) is of very different nature compared to (1.1). It was proved (see [5]) that any nontrivial radial solution to $\Delta u=u^{-p}-1$ in $\mathbb{R}^{n}$ oscillates infinitely many times around the value 1 as $r \rightarrow \infty$ whenever $p>0$ and $n \geq 2$. This suggests that all solutions to (1.3) have infinite Morse index, which is confirmed as follows.

Theorem 1.4 For any $n \geq 2, p>0$ and $R>0$, there are no solutions of (1.3) with finite Morse index. 
The notion of finite Morse index for a solution $u$ of (1.3) is similar as for (1.1), more precisely, it is required that there is only a finite dimensional vector space $X \subset C_{c}^{1}\left(\mathbb{R}^{n} \backslash B(0, R)\right)$ such that

$$
\int_{\mathbb{R}^{n} \backslash B(0, R)}|\nabla \varphi|^{2} d x-p \int_{\mathbb{R}^{n} \backslash B(0, R)} u^{-p-1} \varphi^{2} d x<0, \quad \text { for all } \varphi \in X \backslash\{0\} .
$$

Theorem 1.4 here generalizes Theorems 1.1 and 1.3 in [4].

In the following, the symbol $C$ or $C_{i}, C^{\prime}$ means always a generic positive constant.

\section{Proof of Theorem 1.1}

Suppose that $u$, a solution with finite Morse index to (1.1) exists with some $R>0$. So it is stable outside a compact set, from the stability and (1.1), it is known by Proposition 1 in [1] that

$$
\int_{\mathbb{R}^{n} \backslash B\left(0, R_{0}\right)}|x|^{\alpha} u^{\gamma-p} \psi^{2 m} \leq C \int_{\mathbb{R}^{n} \backslash B\left(0, R_{0}\right)}|x|^{\frac{(\gamma+1) \alpha}{p+1}}\left(|\nabla \psi|^{2}+|\psi \Delta \psi|\right)^{\frac{p-\gamma}{p+1}}
$$

for all $\psi \in C_{c}^{\infty}\left(B\left(0, R_{0}\right)^{c}\right)$ verifying $|\psi| \leq 1$. Here $m \geq \max \left(2, \frac{p-\gamma}{p+1}\right), \gamma \in\left(\gamma_{p},-1\right]$ and

$$
\gamma_{p}=-1-2 p-2 \sqrt{p(p+1)}
$$

the radius $R_{0}>R$ is chosen such that the solution $u$ is stable outside $B\left(0, R_{0}\right)$.

Let $|y| \geq 4 R_{0}$ and $R_{1}=\frac{|y|}{4}$, as $B\left(y, 2 R_{1}\right) \subset B\left(0, R_{0}\right)^{c}$, using (2.1) with standard cut-off function, we have

$$
|y|^{\alpha} \int_{B\left(y, R_{1}\right)} u^{\gamma-p} \leq C|y|^{\frac{(\gamma+1) \alpha}{p+1}} R_{1}^{n-2 \frac{p-\gamma}{p+1}}
$$

hence

$$
\int_{B\left(y, \frac{|y|}{4}\right)} u^{\gamma-p} \leq C|y|^{n-\frac{(2+\alpha)(p-\gamma)}{p+1}}, \quad \forall|y| \geq 4 R_{0}, \gamma \in\left(\gamma_{p},-1\right] .
$$

We write now the equation (1.1) in polar variables

$$
u_{r r}+\frac{n-1}{r} u_{r}+\frac{1}{r^{2}} \Delta_{S^{n-1}} u=r^{\alpha} u^{-p}
$$

and integrate on $S^{n-1}$ to obtain

$$
r^{1-n}\left(r^{n-1} \bar{u}^{\prime}\right)^{\prime}=r^{\alpha} g(r)
$$

where

$$
\bar{u}(r)=\int_{S^{n-1}} u(r, \sigma) d \sigma, \quad g(r)=\int_{S^{n-1}} u(r, \sigma)^{-p} d \sigma .
$$

Integration yields, for all $r>r_{1}>R$,

$$
\bar{u}(r)=\bar{u}\left(r_{1}\right)+r_{1}^{n-1} \bar{u}^{\prime}\left(r_{1}\right) \int_{r_{1}}^{r} t^{1-n} d t+\int_{r_{1}}^{r} t^{1-n} \int_{r_{1}}^{t} s^{n-1+\alpha} g(s) d s d t .
$$

From (2.2) and Hölder's inequality, there holds, for $|y| \geq 4 R_{0}$,

$$
\int_{B\left(y, \frac{|y|}{4}\right)} u^{-p} \leq C|y|^{n-\frac{(2+\alpha) p}{p+1}} .
$$


By a covering argument, this implies

$$
\int_{B(0,2 r) \backslash B(0, r)} u^{-p} \leq C r^{n-\frac{(2+\alpha) p}{p+1}} \text { for } r \geq 4 R_{0},
$$

or equivalently

$$
\int_{r}^{2 r} s^{n-1} g(s) d s \leq C r^{n-\frac{(2+\alpha) p}{p+1}}, \quad \forall r \geq 4 R_{0} .
$$

The dyadic decomposition of the interval $\left[4 R_{0}, r\right)$ gives the following estimate

$$
\int_{4 R_{0}}^{r} s^{n-1+\alpha} g(s) d s \leq C r^{n-\frac{(2+\alpha) p}{p+1}+\alpha} \quad \text { for all } r \geq 4 R_{0}
$$

where we used

$$
n-\frac{(2+\alpha) p}{p+1}+\alpha=n-2+\frac{2+\alpha}{p+1}>0
$$

Remark that for $n \geq 2$,

$$
\int_{r_{1}}^{r} t^{1-n} d t=o\left(r^{\frac{2+\alpha}{p+1}}\right) \quad \text { as } r \rightarrow \infty .
$$

Combining (2.5) with (2.3), we have

$$
\bar{u}(r) \leq C r^{\frac{2+\alpha}{p+1}} \quad \text { for all } r \geq 4 R_{0},
$$

which leads to

$$
\int_{B\left(y, \frac{|y|}{4}\right)} u \leq \int_{B(0,2|y|) \backslash B\left(0, \frac{|y|}{2}\right)} u=\int_{\frac{|y|}{2}}^{2|y|} s^{n-1} \bar{u}(s) d s \leq C|y|^{n+\frac{2+\alpha}{p+1}}
$$

for all $|y| \geq 8 R_{0}$. Since $u$ is subharmonic, we get directly

$$
u(y) \leq C|y|^{\frac{2+\alpha}{p+1}}, \quad \forall|y| \geq 8 R_{0} .
$$

This implies, for any $\gamma<0$,

$$
u^{\gamma-p}(y) \geq C_{1}|y|^{\frac{2+\alpha}{p+1}(\gamma-p)}, \quad \forall|y| \geq 8 R_{0} .
$$

where $C_{1}>0$ is a fixed constant depending on $\gamma$.

Furthermore, we know that (see [1]) the unique solution $\gamma$ to

$$
n+\alpha+\frac{2+\alpha}{p+1}(\gamma-p)=n+\frac{(\gamma+1) \alpha}{p+1}-2 \frac{p-\gamma}{p+1}=0
$$

belongs to $\left(\gamma_{p},-1\right]$ if and only if $p>p_{c}(\alpha)$. Let $\gamma$ satisfy (2.7). We deduce that, thanks to (2.6),

$$
\int_{B(0, r) \backslash B\left(0,8 R_{0}\right)}|x|^{\alpha} u^{\gamma-p} \geq C \int_{8 R_{0}}^{r} \frac{d s}{s} \geq C \ln r-C_{2} \quad \text { for all } r \geq 8 R_{0} .
$$

However, (2.1) with an appropriate test function (see $[3,1])$ gives that, for $\gamma$ verifying $(2.7)$,

$$
\begin{aligned}
\int_{B(0, r) \backslash B\left(0,8 R_{0}\right)}|x|^{\alpha} u^{\gamma-p} & \leq \int_{\mathbb{R}^{n} \backslash B\left(0, R_{0}\right)}|x|^{\alpha} u^{\gamma-p} \psi^{2 m} \\
& \leq C_{3}\left(1+r^{n+\frac{(\gamma+1) \alpha}{p+1}-\frac{2(p-\gamma)}{p+1}}\right)=2 C_{3}<\infty
\end{aligned}
$$

with $C_{3}$ independent of $r$. The estimates (2.8) and (2.9) are clearly in contradiction and show that $u$ cannot be stable outside any compact set. 


\section{$3 \quad$ Proof of Theorem 1.2 and 1.3}

The main idea is very similar to the previous proof, so we just show the essential arguments and omit some details. Suppose that $u>0$ satisfying $\Delta u=|x|^{\alpha} u^{-p}$ in $B(0, R) \backslash\{0\}$ has finite Morse index and a rupture at the origin. There exists then $R_{0}>0$ small such that $u$ is stable in $B\left(0,4 R_{0}\right) \backslash\{0\}$. We can claim

$$
\int_{\left\{0<|x|<4 R_{0}\right\}}|x|^{\alpha} u^{\gamma-p} \psi^{2 m} \leq C \int_{\left\{0<|x|<4 R_{0}\right\}}|x|^{\frac{(\gamma+1) \alpha}{p+1}}\left(|\nabla \psi|^{2}+|\psi \Delta \psi|\right)^{\frac{p-\gamma}{p+1}}
$$

for all $\psi \in C_{c}^{\infty}\left(B\left(0,4 R_{0}\right) \backslash\{0\}\right)$ verifying $|\psi| \leq 1, \gamma \in\left(\gamma_{p},-1\right]$ where $\gamma_{p}=-1-2 p-2 \sqrt{p(p+1)}$ and $m$ large enough.

Taking suitable cut-off function, there holds, by estimates (2.2) and (2.3) in [1],

$$
\int_{\left\{r \leq|x| \leq 2 R_{0}\right\}}|x|^{\alpha} u^{\gamma-p} \leq C\left(1+r^{n+\alpha+\frac{2+\alpha}{p+1}(\gamma-p)}\right), \quad \forall r \in\left(0,2 R_{0}\right), \gamma \in\left(\gamma_{p},-1\right]
$$

and

$$
\int_{B\left(y, \frac{|y|}{4}\right)} u^{\gamma-p} \leq C|y|^{n-\frac{(2+\alpha)(p-\gamma)}{p+1}}, \quad \forall 0<|y| \leq 2 R_{0}, \gamma \in\left(\gamma_{p},-1\right] .
$$

Define $\bar{u}$ and $g(r)$ as above, the estimate (3.3) associated to the covering argument gives

$$
\int_{r}^{2 r} s^{n-1} g(s) d s=\int_{B(0,2 r) \backslash B(0, r)} u^{-p} \leq C r^{n-\frac{(2+\alpha) p}{p+1}} \quad \text { for } 0<r \leq R_{0} .
$$

Using dyadic decomposition of $(0, r)$, we have

$$
\int_{0}^{r} s^{n-1+\alpha} g(s) d s \leq C r^{n-\frac{(2+\alpha) p}{p+1}+\alpha} \quad \text { for all } r \leq 2 R_{0} .
$$

As $r^{1-n}\left(r^{n-1} \bar{u}^{\prime}\right)^{\prime}=r^{\alpha} g(r) \geq 0, r^{n-1} \bar{u}^{\prime}(r)$ is nondecreasing in $r$, we claim that

$$
\ell=\lim _{r \rightarrow 0} r^{n-1} \bar{u}^{\prime}(r)=0 .
$$

Indeed, $\ell \in[-\infty, \infty)$ exists by monotonicity of $r^{n-1} \bar{u}^{\prime}$. As $\lim _{s \rightarrow 0} \bar{u}(s)=0$ by the rupture assumption on $u, \ell \neq 0$ will lead to a contradiction, since $r^{1-n}$ is not integrable at 0 for $n \geq 2$.

Integrating $\left(r^{n-1} \bar{u}^{\prime}\right)^{\prime}=r^{n-1+\alpha} g(r)$, by (3.4) and (3.5), there holds

$$
r^{n-1} \bar{u}^{\prime}(r) \leq C r^{n-\frac{(2+\alpha) p}{p+1}+\alpha}, \quad \text { if } 0<r \leq 2 R_{0},
$$

Then, for any $r>0$, we see that

$$
\lim _{s \rightarrow 0} s^{n-1} \bar{u}^{\prime}(s) \int_{s}^{r} t^{1-n} d t=0, \quad \text { because } 2+\alpha-\frac{(2+\alpha) p}{p+1}=\frac{2+\alpha}{p+1}>0 .
$$

Combining with $\lim _{s \rightarrow 0} \bar{u}(s)=0$ and (3.4), tending $r_{1}$ to 0 in (2.3), we get

$$
\bar{u}(r) \leq C r^{\frac{2+\alpha}{p+1}}, \quad \text { for all } 0<r<2 R_{0} .
$$

Using the fact that $u$ is subharmonic, we can conclude then

$$
u(y) \leq C|y|^{\frac{2+\alpha}{p+1}}, \quad \text { for all } 0<|y|<R_{0} .
$$


If $p>p_{c}(\alpha)$, fix $\gamma \in\left(\gamma_{p},-1\right]$ verify (2.7). The inequality (3.2) implies (tending $r$ to 0 )

$$
\int_{\left\{0<|x| \leq 2 R_{0}\right\}}|x|^{\alpha} u^{\gamma-p}=\int_{0}^{2 R_{0}} s^{n-1+\alpha} g(s) d s<\infty .
$$

However, by estimate (3.6), we see that

$$
\int_{\left\{0<|x| \leq 2 R_{0}\right\}}|x|^{\alpha} u^{\gamma-p}=\int_{0}^{2 R_{0}} s^{n-1+\alpha} g(s) d s \geq C_{1} \int_{0}^{2 R_{0}} \frac{d s}{s}=\infty,
$$

this is absurd. So such a solution with the rupture at zero cannot exist whenever $p>p_{c}(\alpha)$, the proof of Theorem 1.2 is completed.

Finally, Theorem 1.3 is just a direct consequence of the estimate (3.6) to finite Morse index solution with isolated rupture at 0 , which is valid for any $p>0$ and $\alpha>-2$.

\section{Proof of Theorem 1.4}

We argue always by contradiction. Suppose that a solution $u$ with finite Morse index to (1.3) exists. By very similar argument for (2.1), it is showed that (see estimate (2.1) in [4])

$$
\int_{\mathbb{R}^{n} \backslash B\left(0, R_{0}\right)} u^{\gamma-p} \psi^{2 m} \leq C \int_{\mathbb{R}^{n} \backslash B\left(0, R_{0}\right)}\left(|\nabla \psi|^{2}+|\psi \Delta \psi|\right)^{\frac{p-\gamma}{p+1}}
$$

for all $\psi \in C_{c}^{\infty}\left(B\left(0, R_{0}\right)^{c}\right)$ satisfying $|\psi| \leq 1, \gamma \in\left(\gamma_{p},-1\right]$. Here again, $\gamma_{p}=-1-2 p-2 \sqrt{p(p+1)}$ and $R_{0}>R$ is chosen such that the solution $u$ is stable outside $B\left(0, R_{0}\right)$. We can proceed as above to get the corresponding estimates of (2.1), (2.4) with $\alpha=0$, that is,

$$
\int_{B(0,2 r) \backslash B(0, r)} u^{-p} \leq C r^{n-\frac{2 p}{p+1}} \quad \text { for } r \geq 4 R_{0} .
$$

As $n-\frac{2 p}{p+1}>0$ for $n \geq 2$ and $p>0$, the dyadic decomposition argument leads to the following estimate:

$$
\int_{B(0, r) \backslash B\left(0,4 R_{0}\right)} u^{-p} \leq C\left(1+r^{n-\frac{2 p}{p+1}}\right) \leq C^{\prime} r^{n-\frac{2 p}{p+1}} \quad \text { for any } r \geq 4 R_{0} .
$$

On the other hand, integrating equation (1.3) over $B(0, r) \backslash B\left(0,4 R_{0}\right)$, we have

$$
\int_{\partial B(0, r)} \frac{\partial u}{\partial \nu} d \sigma-C=\int_{B(0, r) \backslash B\left(0,4 R_{0}\right)}\left(u^{-p}-1\right), \quad \forall r \geq 4 R_{0} .
$$

Applying (4.2), there holds

$$
\int_{\partial B(0, r)} \frac{\partial u}{\partial \nu} d \sigma \leq C r^{n}\left(r^{-n}+r^{-\frac{2 p}{p+1}}-C_{4}\right), \quad \forall r \geq 4 R_{0} .
$$

Define $\bar{u}$ as before, then

$$
\bar{u}^{\prime}(s)=\int_{S^{n-1}} \frac{\partial u}{\partial r}(s, \sigma) d \sigma=r^{1-n} \int_{\partial B(0, r)} \frac{\partial u}{\partial \nu} d \sigma .
$$

Combining the above two formula, for $s$ large enough,

$$
\bar{u}^{\prime}(s) \leq C s\left(s^{-n}+s^{-\frac{2 p}{p+1}}-C_{4}\right) \leq-C_{5} s,
$$

which implies then $\lim _{r \rightarrow \infty} \bar{u}(r)=-\infty$. This is just impossible since $\bar{u}>0$. 


\section{References}

[1] Y. Du and Z. Guo, Positive solutions of an elliptic equation with negative exponent: stability and critical power, J. Differential Equa. 246(6) (2009), 2387-2414.

[2] P. Esposito, N. Ghoussoub and Y. Guo, Mathematical analysis of partial differential equations modeling electrostatic MEMS, Courant Lecture Notes in Mathematics 20 (2010), American Mathematical Society.

[3] A. Farina, On the classification of solutions of the Lane-Emden equation on unbounded domains of $\mathbb{R}^{N}$, J. Math. Pures Appl. 87(5) (2007), 537-561.

[4] Z. Guo and L. Ma, Finite Morse index steady states of van der Waals force driven thin film equations, J. Math. Anal. Appl. 368(2) (2010), 559-572.

[5] Z. Guo, D. Ye and F. Zhou, Existence of singular positive solutions for some semilinear elliptic equations, Pacific J. Math. 236(1) (2008), 57-71.

Juan Dávila - Departamento de Ingeniería Matemática and CMM (UMI 2807 CNRS), Universidad de Chile, Casilla 170 Correo 3, Santiago, Chile.

E-mail address: jdavila@dim.uchile.cl

Dong Ye - Département de Mathématiques, UMR 7122, Université de Lorraine, site Metz, Ile de Saulcy, 57045 Metz, France.

E-mail address: dong.ye@univ-metz.fr 